\title{
Análisis del concepto de equidad educativa a la luz del enfoque de las capacidades de Amartya Sen
}

\section{Analysis of the concept of educational equity from the Amartya Sen capabilities standpoint}

\author{
María Marta Formichella \\ Docente del Departamento de Economía \\ Universidad Nacional del Sur \\ Buenos Aires, Argentina \\ mfornichella@uns.edu.ar
}

Recibido: 23-VIII-2010 • Aceptado 16-IX-2010 • Corregido 05-IV-2011

\begin{abstract}
Resumen: El objetivo de este trabajo es analizar el concepto de equidad educativa desde la perspectiva de las capacidades propuesta por Amartya Sen. Para ello, en primer lugar se realiza una revisión de la literatura sobre la noción de equidad y se plantea una discusión filosófica al respecto. En segundo lugar, se lleva dicha discusión hacia el ámbito de la educación. En tercer lugar, en concordancia con la aproximación de Sen, se define el concepto de equidad educativa como igualdad en los resultados en las habilidades que adquieren los individuos luego de participar en el sistema educativo (nivel medio). Finalmente, se concluye que bajo esta concepción es posible recomendar políticas para compensar las diferencias de origen.
\end{abstract}

Palabras clave: Equidad, educación, capacidades.

\section{Introducción}

El objetivo de este trabajo es analizar el concepto de equidad educativa desde la perspectiva de las capacidades propuesta por Amartya Sen. Para cumplirlo, en primer lugar se analiza la noción de equidad desde un punto de vista filosófico. En segundo lugar, se aplica dicha noción al ámbito de la educación. En tercer lugar, en concordancia con la aproximación de Sen, se define equidad educativa como igualdad en los resultados en las competencias y habilidades que adquieren los individuos al educarse. Finalmente se presentan las conclusiones del trabajo.

Por un lado, en la literatura económica existe un consenso con respecto a que la educación es determinante para el crecimiento económico. Algunos autores 


\begin{abstract}
The purpose of this research is to analyze the concept of educational equity from the capabilities standpoint proposed by Amartya Sen. To do this, first the literature on the notion of equity is reviewed and a philosophical discussion about it is presented. Second, it is applied to the educational field. Third, according to the Sen approach, educational equity is defined as equality in outcomes in abilities that individuals aquire in the educational system (secondary level). Finally, it is concluded that under this conception it is possible recommend policies to compensate original differences.
\end{abstract}

Keywords: Equity, education, capabilities. dan importancia al stock de capital humano acumulado (Nelson y Phelps 1966, citados en Aghion y Howitt 1998), mientras que otros destacan el rol de la tasa de crecimiento de capital humano (Lucas, 1988).

Asimismo, Thomas, Vinod, Wang, Yan y Fan y Xibo (2002) afirman que es importante medir la distribución de capital humano y no solo su nivel promedio o su tasa de crecimiento, argumentando su postura con base en aspectos vinculadas al bienestar y a la eficiencia.

En relación al bienestar, estos autores citan a Sen y expresan que la educación mejora la capacidad de las personas, es decir, aumenta las libertades fundamentales que estas poseen para optar por el estilo de vida que deseen llevar adelante. En este sentido, la educación es propicia para el desarrollo (Sen, 1999a). Así, refieren a que si la pobreza es una privación de capacidades elementales, las medidas de inequidad en el bienestar deben incluir medidas de inequidad en la educación.

Con respecto a la eficiencia, Thomas et ál. (2002) dicen que el crecimiento se ve afectado por la distribución de los activos y que el capital humano es uno de los más relevantes; por esto, estudiar su distribución aparece como primordial. De hecho, estos autores realizan un análisis empírico y encuentran una relación negativa entre desigual distribución de años de escolarización y crecimiento. De igual modo, otros autores también han hallado evidencia de que existe una correlación negativa entre el nivel de dispersión de la educación y la tasa de crecimiento (Birdsall y Lodoño, 1997; Castello y Doménech, 2002; López, Thomas y Wang, 1998).

Asimismo,importantes autores argentinos han hecho hincapié en la necesidad de abordar esta temática argumentando que la equidad educativa puede aumentar la equidad económica (Gasparini, 2001, Guadagni, 2007).

Por último, cabe mencionar que diversos pensadores (Cohen, 1989; Roemer, 1995; Sen, 1979) consideran que 
las oportunidades de las personas deben ser iguales para que la sociedad sea justa, y la educación no es una cuestión menor a la hora de analizar las potencialidades de desarrollo de un individuo (Sen, 1999a).

\section{El concepto de equidad}

El concepto de equidad es sumamente complejo y sus definiciones han ido variando a través del tiempo. D’Lia y Maingon (2004) señalan que las teorías que han estudiado el tema están de acuerdo en vincularlo a las diferencias relacionadas a condiciones de injusticia. Ya Aristóteles en su libro "Moral a Nicómano" establecía que, si bien equidad y justicia no son conceptos idénticos, poseen la misma esencia (Azcárate Corral, 1874).

Asimismo, Rawls (1999) expresa que la idea de equidad es fundamental en el concepto de justicia y destaca que los miembros de una sociedad discuten y establecen qué prácticas ${ }^{1}$ definirán como justas, lo cual implica que las mismas dependerán del pacto subyacente entre los individuos pertenecientes a una comunidad.

Por ello, si bien la palabra equidad proviene del latín aequitas, que significa "igual”, en función de cuál sea el aspecto (base de información en palabras de Sen, 1979) que se considere relevante tomar en cuenta para igualar a las personas, existen diferentes teorías de la justicia social. Así, Dworkin (1981) plantea que la equidad es una idea política popular, pero misteriosa. Las personas pueden ser iguales en un sentido, pero no en otro, sin que esto implique que la equidad sea un concepto inútil. Lo relevante es analizar bajo qué forma la misma será contemplada.

Entonces, equidad quiere decir igualdad en cierto atributo, lo cual no implica igualdad en todos los atributos. Para que esto fuera así no debería existir ninguna clase de diferencia entre los individuos, lo cual no coincide con la realidad en absoluto. Es más, para lograr la igualdad en un aspecto puede ser necesario que exista desigualdad en otro (Sen 1995, citado en López, 2006).

Sen (1979), ante la expectativa de definir el concepto de equidad, plantea el interrogante: ¿igualdad de qué? Las posibles respuestas a esta pregunta pueden resumirse de la siguiente manera (en las próximas líneas se explicará cada una):

Igualdad en el bienestar o "welfarismo": aquí se incluyen las diferentes versiones de análisis del utilitarismo.

Igualdad en los bienes: abarca la concepción de "bienes primarios" de Rawls y de "recursos" de Dworkin (Fleurbaey, 1995).

Igualdad en las oportunidades: aquí se hace referencia a la idea de "capacidades básicas" de Sen, de "alisar el camino" de Roemer, de "oportunidades para el bienestar" de Anerson y de "acceso a las ventajas" de Cohen (Fleurbaey, 1995).

El "welfarismo" plantea que el bienestar es juzgado en términos de utilidad. Se supone que la utilidad representa una medida del placer o felicidad de las personas y, dadas las dificultades que representa su medición, esta se define como una representación numérica de las elecciones observables de las personas. Según este enfoque, el objetivo es maximizar la suma total de utilidad, independientemente de su distribución; por lo tanto, una sociedad es injusta si sus miembros considerados en forma conjunta son menos felices de lo que podrían ser (Sen, 1979; 1999a).

Sen (1979) cuestiona el uso de la utilidad como medida del bienestar porque esta perspectiva no le da relevancia a los derechos y libertades, sino que los pondera indirectamente por su efecto sobre las utilidades. Según él, un esclavo puede ser feliz, pero no tiene por qué carecer de libertad.

Además, este autor expresa que centrar la atención en condiciones de la mente, como el placer o los deseos, puede no ser apropiado cuando se intentan realizar comparaciones interpersonales de bienestar, ya que los deseos y las capacidades para disfrutar de las circunstancias se 
adaptan a las condiciones de vida de los individuos (Sen, 1999a) ${ }^{2}$.

Otra crítica a la concepción "welfarista" de la equidad tiene que ver con la idea de los "gustos caros". Rawls (1986, en Hernández, 2006) afirma que las preferencias no son apropiadas para analizar la justicia social y sostiene que la visión utilitarista es "ciega" respecto de la responsabilidad que tienen los individuos sobre sus preferencias. En este sentido, cabe preguntarse si es justo compensar las diferencias de utilidad interpersonales cuando las mismas provienen de la existencia de individuos con preferencias "caras".

Respecto a la igualdad en los bienes, Rawls (1971) plantea que cada persona posee su libertad y que la misma no puede verse atropellada por ningún motivo, ni siquiera en pos de aumentar el bienestar general. En este sentido, Caballero (2006) expresa que la teoría de la justicia de Rawls aparece como reacción al utilitarismo, siendo su principal crítica hacia este el hecho de que "la utilidad individual se vea diluida en un océano de utilidad global".

Rawls considera que los principios de justicia social serían aquellos que las personas libres y racionales aceptarían si estuvieran en una situación inicial de igualdad. Mendioca y Veneranda (1999) destacan que en dicha "posición original" no se conoce quiénes serán los favorecidos, ni cuáles serán las preferencias posteriores, ni cuál es el curso de acción futuro en la comunidad. En ese momento, simplemente, todos son racionales y capaces de tomar decisiones según su moral. Dichas decisiones serán justas debido a las condiciones de igualdad en las que se toman.

Bajo este contexto, Rawls (1971) analiza la igualdad en función de lo que él denomina "bienes primarios". Estos son aquellos que se supone que el hombre racional desea; incluyen derechos, libertades y oportunidades; ingreso y riqueza; y las bases sociales del respeto a sí mismo.

Por otro lado, Dworkin (1981) propone definir a la equidad como igualdad en los recursos materiales. Esto podría asemejarse a cierto tipo de "igualdad en las oportunidades", las cuales estarían representadas por los recursos. Según este autor, existirá igualdad en los recursos cuando cada individuo esté satisfecho con su "cesta" de bienes y no prefiera la cesta de recursos de ningún otro; en dicho momento se habrá superado el "test de la envidia".

Por último, en relación a los autores que proponen la igualdad en las oportunidades, Sen (1979) presenta una nueva manera de definir la equidad. Según él mismo afirma, su visión puede entenderse como una extensión de la idea de justicia de Rawls. Sen parte del problema que implica usar a los "bienes primarios" como base de información. Dicho problema se evidencia en que, aunque los bienes pudieran ser igualados, no se solucionarían las desventajas. Esto porque los seres humanos son diferentes y existe una gran variabilidad en lo que ellos son capaces de hacer con su cesta de "bienes primarios", por lo que requieren de diferentes cantidades de estos para satisfacer las mismas necesidades. Por ello, Sen agrega a la propuesta de los "bienes primarios" las discrepancias que poseen las personas para explotar las circunstancias y oportunidades con las que azarosamente se encuentran en la vida, y su planteamiento puede verse como una extensión del de Rawls.

En el análisis de Sen $(1979,1999 a)$ la base de información son las "capacidades". La capacidad de una persona se define como las distintas combinaciones de funciones que esta puede llegar a lograr. El concepto de funciones tiene un origen aristotélico y refleja las diferentes cosas que un individuo puede valorar hacer o ser. Entonces, la capacidad es un tipo de libertad: la libertad fundamental para lograr combinaciones de funciones, es decir, diferentes estilos de vida. En este contexto, interesa el aspecto de "agencia" de los individuos. Se define a la persona como agente en el sentido más antiguo de la palabra, es decir, como aquel que actúa, provoca cambios y alcanza 
objetivos que pueden evaluarse en relación a sus propios juicios de valor, más allá de que también pueda o no existir una mirada externa.

Como puede observarse, en el enfoque de Sen, el centro está en las libertades que generan los bienes y no los bienes en sí mismos. Aquí, el criterio igualador son las "libertades fundamentales" o "capacidades" (capabilities) que tienen las personas para elegir su modo de vida, al cual valoran y tienen motivos para valorar. El éxito de una sociedad se evalúa, desde esta perspectiva, con relación a las libertades fundamentales de que disfrutan sus miembros. En este sentido, si dos personas se encuentran en idéntica situación, eso no significa que la misma sea equitativa, porque puede ser que una haya optado por dicha situación, mientras que la otra no (Sen 1979, 1999a).

Por su parte, Roemer (1995) también plantea que la igualdad tiene que estar dada en las oportunidades. Considera que los resultados son consecuencia de las acciones humanas, pero pone en el centro de su análisis el hecho de que dichas acciones están determinadas por dos tipos de causas: circunstancias controladas por los individuos y circunstancias que están fuera del alcance de los mismos.

De acuerdo con este autor, existe equidad en las oportunidades si las personas que se encuentran desfavorecidas dentro de la distribución de cierto resultado ${ }^{3}$ como consecuencia de circunstancias que quedaron afuera de su esfera de decisión, son indemnizadas por el resto de los individuos de la sociedad. Entonces, según Roemer, en una sociedad en la que exista igualdad de oportunidades no habrá ningún ciudadano que esté en una peor situación que otro como consecuencia de factores que estén fuera de su control.

Es difícil de determinar qué factores están dentro del alcance de las personas a la hora en que estas realizan sus elecciones, por ello Roemer sugiere una manera de estudiar esta problemática. Plantea que se debe clasificar a los individuos en grupos de acuerdo a sus características; de esta manera quedarían conformados conjuntos de "tipos" de individuos. Dado que las condiciones son idénticas dentro de cada grupo, las discrepancias en los resultados logrados por parte de los individuos al interior de los mismos se deberán diferenciar en el esfuerzo que cada uno de ellos ha realizado. Entonces, las discrepancias existentes dentro de cada grupo no deberían ser compensadas porque son consecuencias de una elección individual y no se vinculan a circunstancias externas al individuo, sino que pertenecen a la esfera de la responsabilidad personal.

A su vez, si en cada grupo se ordena a las personas de acuerdo a los resultados obtenidos, se puede tener una aproximación del esfuerzo que cada una ha realizado. Entonces, es factible conocer quiénes, a pesar de formar parte de diferentes grupos, han realizado el mismo esfuerzo y compensarlos si obtuvieron un resultado menor.

Otro autor que se preocupó por analizar el tema de la justicia social es Richard Arneson, quien sobre fines de la década del ochenta plantea la idea de la equidad como "igualdad de oportunidades para el bienestar" (Ribotta, 2006, p. 145).

Arneson toma en cuenta las oportunidades porque sostiene que la igualdad de bienestar en si misma es pobre. En la igualdad de bienestar, los bienes se repartirían entre las personas de manera que cada una pudiera disfrutar del mismo nivel. Sin embargo, las personas podrían llegar a diferentes resultados en función de las decisiones que tomaran, aunque su punto de partida fuera el mismo. Por ello, este autor considera que para que exista equidad es necesaria la igualdad de "oportunidades para el bienestar" (potencial), lo que significa que cada individuo tiene derecho a poseer las mismas opciones que el resto con relación a su perspectiva de satisfacción de preferencias. Entonces, solo se puede alcanzar la igualdad de oportunidades para el bienestar si cada persona, al llegar a la vida adulta, accede a un conjunto de alternativas realmente igual 
al de las demás. Además, la sociedad tiene la obligación de compensar las diferencias entre las personas menos habilidosas para elegir porque la igualdad de oportunidades para el bienestar tiene que garantizar que todos aquellos que hagan un mismo esfuerzo puedan lograr obtener una expectativa de bienestar equivalente (Ribotta, 2006).

En este sentido, Arneson explica que las personas pueden ser diferentes en su capacidad para reconocer las oportunidades, en su habilidad para tomar decisiones, o incluso en su fortaleza para no abandonar la decisión una vez que ha sido elegido un camino. Entonces, para que la igualdad de oportunidades sea verdaderamente real, es necesario que todos los individuos posean las mismas habilidades para analizar las opciones, que las opciones estén planteadas de manera tal que se puedan anular las diferencias en habilidad, o que las desigualdades en las habilidades de elección sean responsabilidad de las personas. Entonces, existe igualdad en el bienestar cuando los individuos realmente se enfrentan a conjuntos de alternativas equivalentes (Armesto, 2007).

Un autor contemporáneo y complementario a Arneson es Gerald Allan Cohen. Cohen (1989) plantea que hay equidad si existe igualdad en el "acceso a las ventajas". Destaca la relevancia de distinguir entre suerte y elección en la formación de las personas, ya que en pos de la igualdad se considera esencial eliminar la influencia de la suerte en la distribución de resultados. El "acceso a ventajas" significa que debe haber igualdad de oportunidades de obtención de una posición social, pero también y principalmente a la hora de adquirir las cualificaciones necesarias para competir por las diferentes posiciones.

Cohen (1989) diferencia su noción de igualdad de acceso a las ventajas de la idea de igualdad de oportunidades para el bienestar de Arneson afirmando que la idea de ventaja es más amplia que la de bienestar. Este autor manifiesta que la ventaja es un conjunto de estados deseables muy disímiles del individuo, que no se pueden representar únicamente por recursos o por el bienestar, aunque sí los incluye, ya que cuando los recursos y el bienestar son deficientes se está en presencia de una desventaja. La idea de ventaja es parecida a la idea de funciones que propone Sen (Ribotta, 2006).

En síntesis, por un lado existen las propuestas "welfaristas" y por el otro las "post-welfaristas". Las primeras utilizan como base de información alguna medida del bienestar, mientras que las segundas critican esa elección y proponen, cada una, el uso de una alternativa diferente. Asimismo, dentro de las ideas post-welfaristas se encuentran las fundadas en la igualdad de los bienes (Dworkin, 1981; Rawls, 1971) y las que se centran en la igualdad de las oportunidades (Arneson, 1989; Cohen, 1989; Roemer, 1995; Sen, 1979).

Dadas las críticas enunciadas anteriormente acerca del análisis "welfarista", en este trabajo se pondrá atención en las propuestas "post-welfaristas". Asimismo, dado que el centro de análisis es la educación, dentro de estas propuestas interesa estudiar especialmente la igualdad en las oportunidades, ya que los individuos difieren en sus habilidades para sacar provecho de los recursos disponibles a la hora de educarse. Por ende, considerar la igualdad en los recursos no es suficiente para analizar la equidad en el ámbito de la educación ${ }^{4}$.

Dentro del grupo de los autores que toman en consideración la igualdad de oportunidades, pueden realizarse algunas vinculaciones. Como se ha destacado anteriormente, la propuesta de Cohen surge como una ampliación de la de Arneson y, al mismo tiempo, ambas se relacionan estrechamente con la postura de Sen.

Por un lado, el mismo Arneson reconoce que su planteamiento de igualdad en las oportunidades de bienestar es similar a la concepción de igualdad en las capacidades propuesta por Sen. Destaca que la 
manera en que Sen define el bienestar (a partir de las ideas de funcionamientos y capacidades, y de su vínculo con la concepción de libertad como desarrollo) implica que la idea de igualdad de capacidades, no solo se asemeje en gran medida a la de igualdad de oportunidades para el bienestar, sino que también la abarque. Entonces, dentro de la propuesta de Sen estaría incluida la de Arneson (Ribotta, 2006).

Por otro lado, el concepto de ventaja de Cohen se asemeja al concepto de funcionamiento de Sen. Sin embargo, Cohen (1989) entiende que su propuesta es más amplia que la de Sen y critica algunas cuestiones del planteamiento de igualdad en las capacidades. Principalmente, no está de acuerdo en que todos los aspectos de la equidad se vinculen a la idea de capacidad. Si bien reconoce que es cierto que no es lo mismo poseer un bien que tener la capacidad de hacer algo con dicho bien, argumenta que no todo será resultado del ejercicio de la capacidad. En este sentido, Molina (2000) explica que esto significa que en ocasiones los bienes causan estados deseables en los individuos sin que estos tengan que hacer nada.

Es decir, Cohen establece que los bienes benefician a las personas más allá de la reacción mental o evaluación que las personas hagan sobre los mismos. A esto Cohen lo denomina vía media porque de alguna manera se encuentra a mitad de camino entre los bienes y la utilidad, y en base a la vía media objeta el enfoque de las capacidades de Sen. Por su parte, Sen, señala que lo que Cohen nombra como vía media no es lo que él entiende como capacidad, sino que serían los funcionamientos (Ribotta, 2006).

Entonces, la propuesta de Arneson está incluida en la de Sen y, si bien no hay consenso total, la propuesta de Cohen se asemeja en gran medida a la de Sen. Lo relevante es destacar que todos centran su atención en las verdaderas oportunidades que tienen los individuos y se alejan, cada uno con sus argumentos, de la idea que vincula igualdad en recursos o bienes con igualdad en las oportunidades.
Por otra parte, existen otros vínculos a tener en cuenta entre las propuestas postwelfaristas. Ribota (2006) agrupa a las concepciones de Dworkin, Roemer, Cohen y Arneson dentro del denominado "igualitarismo de la suerte"5. Para los igualitaristas de la suerte, la equidad consiste en eliminar en la medida de lo posible el efecto de toda mala suerte en el trayecto de vida de las personas. Entonces, las únicas desigualdades legítimas en una sociedad justa serían las que provienen de elecciones voluntarias de los individuos y, en consecuencia, pertenecen a la esfera de su responsabilidad. El resto de las diferencias deberían ser compensadas.

Más allá de esta agrupación, cabe señalar que Cohen, Roemer y Arneson critican a Dworkin por considerar que las preferencias individuales forman parte de la responsabilidad personal (Maldonado y Ríos, 2006). En este sentido, Igersheim (2006) explica que la literatura clásica (representada principalmente por Fleurbaey) clasifica a los autores en relación a la responsabilidad de la siguiente manera: por un lado, los que consideran que existe responsabilidad individual por las preferencias -incluye a Rawls (1971) y Dworkin (1981) - y, por otra parte, los que tienen en cuenta una mezcla de determinismo con responsabilidad -incluye a Sen (1979), Arneson (1989), Cohen (1989) y Roemer (1995)-. Esta clasificación es coincidente con la realizada según la base de información utilizada para definir equidad: el primer grupo se corresponde con los autores que defienden la idea de la igualdad de recursos, mientras que el segundo grupo está representado por los autores que se concentran en la igualdad de las oportunidades.

Como puede notarse, ahora Sen se incluye entre los autores que analizan la responsabilidad individual. Esto es así porque si bien en su propuesta este autor no hace explícita su consideración sobre la responsabilidad, no es del todo correcto afirmar que dicha concepción no está presente. En el planteamiento de Sen los logros de 
una persona dependen del vector de funcionamientos que eligió dentro de su conjunto de capacidades. Dicho conjunto está en función de cuestiones de las que el individuo no es responsable, pero sí es responsable por los bienes y funciones de utilización de estos que elija (Igersheim, 2006).

Por último, siguiendo a Igersheim (2006) se observa que existe un vínculo entre las ideas de Roemer y Sen ${ }^{6}$. Por una lado, la literatura clásica encasilla a estos dos autores dentro de una misma categoría; por otro lado, Roemer se auto-define diferente a Sen y critica la poca profundización y claridad que le otorga al tema de la responsabilidad. Más allá de esto, lo cierto es que, aunque no en todos los aspectos, en algunos puntos relevantes estos pensadores coinciden.

Ambos autores, basándose en la idea de la diversidad humana, consideran la existencia de determinismo en el comportamiento, no imputándoles la totalidad de la responsabilidad de sus preferencias a las personas. Sin embargo, difieren en el "justice cut"7, para Roemer las personas no son responsables de las circunstancias que las llevan a pertenecer a un "tipo", mientras que para Sen los individuos no son responsables de su capacidad. Asimismo, para Roemer hay responsabilidad sobre el grado de esfuerzo, y Sen considera que la responsabilidad está en la elección del vector de funcionamientos. Por ello, difieren en el modo de compensación, ya que en principio lo que se intenta igualar (es decir el atributo por el que se define la equidad) coincide con el elemento sobre el cual las personas no tienen responsabilidad alguna; sin embargo y como se verá en breve, Roemer da un paso más adelante en dicho sentido.

En su perspectiva, Sen sólo plantea la igualación de las capacidades como método de compensación. Por su parte, Roemer recomienda igualar el conjunto de oportunidades de todos los individuos, pero también avanza más y dice que hay que equiparar los resultados de quienes realizaron el mismo grado de esfuerzo. Su clasificación de los individuos por "tipo" y el hecho de que la distribución de resultados sea una característica de cada grupo hacen que esto sea posible y ahí radica su mayor aporte. No obstante, esto acerca la teoría de Roemer a una teoría de equidad en los resultados, más que a una de las oportunidades. En este sentido, Roemer propone métodos más concretos de compensación, mientras que la teoría de Sen se focaliza más en prevenir las inequidades y establecer cómo sería una sociedad justa. Por ello esta última se acerca más a una teoría de igualación de las oportunidades.

En síntesis, en este apartado se han descrito las principales ideas referidas a la equidad social y se han analizado algunas de las relaciones que presentan. El propósito es ahora avanzar un paso más y vincular estas ideas con el fenómeno de la educación, para poder definir así a la equidad educativa.

\section{El concepto de equidad aplicado al ámbito de la educación}

Al examinar la concepción de equidad vinculada al fenómeno de la educación surgen dos cuestiones: por un lado, la educación puede analizarse como un elemento necesario para que se logre la equidad social y, por otra parte, es menester estudiar qué implica la existencia de equidad en el ámbito educativo.

Con respecto al primer punto, y en función del análisis presentado en la sección anterior, puede deducirse que la educación adquiere un papel central en todas las visiones: en el caso del paradigma "welfarista" la educación puede verse como un elemento que hace aumentar la utilidad de los individuos; por su parte, en los enfoques que proponen la igualdad en los bienes o recursos, la educación puede pensarse como un activo más a ser igualado entre los individuos; por último, en relación con las ideas referidas a la igualdad en las oportunidades, dado 
el rol que tiene la educación en el desarrollo de las personas, queda claro que la misma es un componente necesario para hacer que estas se encuentren en un mismo punto de partida (cierto nivel de educación mínimo es requerido para equiparar las oportunidades).

El análisis que realiza Sen es particularmente interesante con relación a las oportunidades. De acuerdo a la noción de equidad propuesta por Sen, en la que la base de información son las libertades fundamentales que poseen las personas para optar por un estilo de vida propio, la educación adquiere un papel central.

Sen (1999a) plantea la existencia de un grupo de libertades a las que denomina instrumentales, ya que contribuyen directa o indirectamente a que los individuos puedan vivir del modo que desean. Entre dichas libertades enumera: a) las libertades políticas, b) los servicios económicos, c) las oportunidades sociales, d) las garantías de transparencia y e) la seguridad protecto$\mathrm{ra}^{8}$. Cuando menciona las oportunidades sociales hace referencia, entre otros, a los sistemas educativos que influyen en cómo podrán vivir su vida los individuos. La educación afecta su vida privada y también sus oportunidades de socializarse y participar con mayor eficacia en las actividades económicas y políticas.

En relación al segundo punto, es decir a qué implica la equidad en el ámbito educativo, la pregunta a responder es: ¿Cuándo existe equidad educativa? Esta interrogante apunta a determinar qué aspecto de la educación se toma en cuenta para analizar su igualdad y, por ende, llegar a una definición de la equidad educativa en función del mismo. Es decir: ¿Cuál es la base de información adecuada para evaluar la equidad educativa?

Como se dejó entrever al estudiar el concepto de equidad en el apartado anterior, qué criterio igualador elegir tiene que ver con consideraciones subjetivas respecto a qué cuestión es más valorada a la hora de igualar a las personas. En este trabajo se elige la concepción de Sen para estudiar la equidad educativa, debido a que este autor se focaliza en cómo prevenir las inequidades. Dado que los individuos en edad escolar son niños o adolescentes, es decir que están atravesando las primeras etapas de sus vidas, la prevención aparece como una herramienta más interesante que la compensación posterior.

Saito (2003) hace una consideración especial con respecto a la posibilidad de adaptar la concepción de equidad basada en las capacidades de Sen a los niños. Destaca que muy pocos negarían que los niños necesitan de sus padres, maestros, adultos de la sociedad en general, para poder elegir cómo vivir sus vidas; entonces, bajo esta premisa la aproximación de las capacidades de Sen no podría aplicarse a individuos que no estuvieran totalmente maduros para tomar decisiones por ellos mismos. En este sentido y con relación a la educación, cita a White (1973), quién afirma que si se le da a un chico la posibilidad de elegir qué cosas aprender y cuáles no, es probable que falle y deje de formarse en cuestiones que necesitará en su vida.

Sin embargo, Sen (1999a) resalta la relevancia de la libertad futura de los individuos y explica que otorgarle libertad a un chico en el presente puede que lo lleve a disponer de menos libertad en el futuro, mientras que restringirle la libertad hoy puede ampliársela en su adultez. Ejemplos de esta propuesta pueden ser el hecho de obligar a un niño a vacunarse o a ir a la escuela, entre otros. Por ello, este autor sugiere estudiar la libertad de los niños en una perspectiva que abarque toda su vida y aclara que la educación debe ser brindada de manera tal que le dé herramientas para ser verdaderamente autónomo en su adultez.

De acuerdo con lo explicado en los párrafos anteriores, sí puede adaptarse el planteamiento de Sen al caso de niños y adolescentes, pero el tratamiento de la responsabilidad individual que se encuentra implícito en su análisis no podría aplicarse, ya que sus decisiones estarían reflejando, 
en realidad, las decisiones de los adultos que están a su cargo. Es decir, los caminos que tomen los niños dependerán totalmente de circunstancias ajenas a ellos, ya que ningún niño elige a los adultos que lo guiarán, ni el medio ambiente en el cual desarrollarse.

Esto difiere del planteamiento que realiza Roemer (1998), quien considera que una vez que se han establecido las compensaciones necesarias para que todos tengan las mismas oportunidades en educación, las diferencias se deberán a discrepancias en los niveles de esfuerzo que cada individuo realiza y no a sus circunstancias. Sin embargo, Bolivar (2005) explica que el problema de esta concepción está en que el propio esfuerzo de los alumnos no es independiente de otros condicionamientos sociales.

Asimismo, hay otros autores que también manifiestan que no puede hablarse de responsabilidad individual no influenciada por las circunstancias en el caso de los adolescentes, ya que estos realizan elecciones en función de diversas motivaciones que tienen que ver con su medio ambiente (Akerlof y Kranton, 2002; Bolivar, 2005).

Los adolescentes, si bien en menor medida que los niños también necesitan ayuda para elegir, como se señaló para el caso de ellos. Los adultos de la sociedad constituyen su guía, especialmente $\mathrm{su}$ familia Montes (2004) explica que la misma forma parte de los referentes ineludibles de los jóvenes y que es la familia la que hace posible o no que ellos accedan a recursos y oportunidades. Además, señala que los contextos mediatos e inmediatos de los adolescentes influyen sobre sus condiciones de posibilidad.

Entonces, el enfoque elegido para estudiar la equidad educativa es el propuesto por Sen. Sin embargo, bajo la aclaración de que el análisis que él hace de la responsabilidad individual no puede ser aplicado en el caso de los niños y adolescentes. Aplicar la propuesta de Sen a la educación significa tomar en cuenta los funcionamientos que los individuos en edad escolar poseen y además estudiar cómo la restricción de su libertad presente puede colaborar para que ellos alcancen resultados exitosos en su carrera de aprendizaje, lo cual les aumentará su libertad futura. A continuación, a la luz del enfoque elegido, se analizan diferentes principios que se han discutido en el ámbito de la equidad educativa.

\section{Equidad educativa a la luz del enfoque de las capacidades de Sen}

Marc Demeuse (citado en López, 2006) plantea que, en el ámbito de la educación, existen cuatro principios de equidad que compiten entre sí: a) igualdad en el acceso, b) igualdad en las condiciones o medios de aprendizaje, c) igualdad en los resultados o logros, e d) igualdad en la realización social de dichos logros.

El primer criterio enunciado se vincula con la igualdad de recursos. Su lógica ética se acerca al planteamiento de Rawls o Dworkin, ya que establece que un sistema educativo es equitativo si todos los individuos poseen las mismas posibilidades de acceder a él. Es decir, si los sistemas educativos brindan condiciones materiales similares a quienes están en edad escolar y el acceso a la educación es libre y gratuito puede decirse que existe equidad. Es una suerte de igualdad en las oportunidades basada en los recursos, lo cual implica que lo que ocurra en la trayectoria de cada individuo forma parte de su responsabilidad.

Como ya se ha mencionado, Sen critica esta forma de definir la equidad, ya que deja de lado el hecho de que los individuos difieren en sus capacidades para convertir los recursos en logros.

Al plantearse la igualdad en el acceso se está considerando equitativo, en palabras de Aristóteles, el "igual tratamiento para los iguales", es decir una especie de equidad horizontal educativa. Los "iguales" en educación serían los individuos que poseen una determinada edad 
(niños o jóvenes) y son potencialmente alumnos (Morduchowicz, 2000a).

Este criterio supone implícitamente que todas las personas llegan en condiciones homogéneas a la escuela. López (2006) resalta que este punto de vista deja afuera las desigualdades que puedan provenir de diferencias sociales y culturales de los alumnos. Morduchowicz (2000a) expresa que el acento está puesto únicamente en que la posibilidad de un niño de asistir a la escuela no dependa de factores ajenos a él (lo que se denomina "libertad negativa"9). Por su parte, Clune (1994) expresa que la utilización de las oportunidades de acceso educativas como base de información es insuficiente, ya que no sólo no considera qué sucede con el uso y la calidad de los insumos, sino que también deja totalmente de lado el proceso enseñanza-aprendizaje.

Una propuesta de equidad basada en este criterio avala la instalación de un sistema meritocrático, lo cual implica que lo que cada individuo logra depende únicamente de su esfuerzo y su talento. De este modo, la escuela es el instrumento para que los individuos se desarrollen en función de sus propias cualidades y quien no llega a la meta es el único responsable de no poder hacerlo. Fenómenos indeseables como el fracaso escolar y la deserción escolar estarían legitimados desde esta lógica. Un sistema educativo basado en la meritocracia no logra acabar con las diferencias iniciales, sino al contrario, estas tienden a profundizarse. Una escuela meritocrática es una escuela que selecciona a los más capaces y productivos, dejando fuera a quienes inician su formación en una situación de desventaja frente al resto (López, 2006).

Este mecanismo evidencia una lógica que, desde la óptica de las capacidades de Sen, puede ser considerada injusta: los individuos menos aventajados fracasan en el contexto de un sistema educativo que no toma en cuenta las diferencias iniciales y limita las libertades fundamentales de este grupo de personas, subyaciendo en forma implícita que la responsabilidad del fracaso es de ellas.
Con respecto al segundo criterio enunciado, la igualdad en los medios, se está haciendo referencia a la igualdad en los procesos de enseñanza. Esto significa tratar de la misma manera a todas las personas que ingresan en el sistema educativo, utilizar las mismas técnicas pedagógicas y las mismas estrategias (López, 2006).

Este principio no tiene en cuenta en absoluto la educabilidad de los individuos. La educabilidad, interpretada en su sentido habitual, hace referencia a las condiciones sociales necesarias para que un individuo pueda ir a la escuela y participar exitosamente de las clases (López, 2006). Dichas condiciones tienen que ver con aspectos de desarrollo cognitivo básico, que se produce en los primeros años de vida y se vincula, por un lado, con la estimulación afectiva, la alimentación y las condiciones sanitarias y, por otro lado, con la socialización primaria de los chicos, la cual los prepara a insertarse en una institución diferente a su familia (Tedesco, 2000).

La noción tradicional de educabilidad se refiere a que, de acuerdo a cómo está organizada la escuela, "se espera" que los alumnos posean ciertas características para poder transitar por ella ${ }^{10}$. Sin embargo, como ya se ha mencionado, los alumnos difieren entre sí y no todos poseen el bagaje de recursos necesario para articular con lo que les ofrece el sistema educativo.

Cabe destacar que este precepto de equidad basado en la igualdad en los medios no solo supone implícitamente que no existen diferencias entre los alumnos, sino que deja totalmente de lado la cuestión del acceso. Según este criterio, la educación de los individuos de una sociedad podría ser definida como equitativa y al mismo tiempo existir altos porcentajes de personas en edad escolar que no asisten al colegio, y altos índices de deserción.

En el contexto de las libertades fundamentales de Sen esto no parecería ser justo, puesto que las personas que quedan afuera de la posibilidad de asistir a la escuela se ven privadas de un tipo 
de libertad instrumental: la oportunidad social. Sen (2003) expresa que no ser capaz de leer, escribir y comunicarse es un caso de privación extrema.

Asimismo, la aplicación de una estrategia uniforme en la enseñanza no parece tomar en cuenta las diferencias en las capacidades (en el sentido de Sen) de los individuos. Los alumnos "no educables" que logren ingresar en el sistema escolar se enfrentarán con serias dificultades que les impedirán alcanzar los resultados deseados.

Con relación al criterio basado en la igualdad en la realización social de los logros, la equidad es evaluada en función del impacto que tiene un sistema educativo en el desarrollo social. Esto podría llegar a justificar que dentro de un mismo país algunas regiones reciban más educación que otras porque así lo necesitan dado su grado de desarrollo. En este caso, la educación es valorada en términos del desarrollo regional que pueda aportar y no cómo un bien en sí mismo (López, 2006).

A la luz del concepto de equidad de Sen, no tomar en cuenta a la educación como un valor en sí mismo y que eso redunde en que individuos de una región reciban menos educación que quienes habitan en otra implica que algunas personas se vean limitadas en su libertad. Por ello, este criterio tampoco parece ser adecuado bajo el enfoque de las capacidades.

Por último, cabe analizar el principio de equidad basado en la igualdad en los resultados. Morduchowicz (2003) explica que la utilización de los resultados educativos como base de información implica que la educación debe ser adecuada a algún propósito, específicamente a los resultados de los alumnos. Sin embargo, este autor aclara que la igualdad en los resultados no contradice ni es incompatible con la igualdad en las oportunidades, sino que se trata de que verdaderamente se establezcan las condiciones y se comprometan los recursos para que todos los individuos tengan la misma oportunidad de arribar a los resultados definidos como deseables.
Además, explica que más allá de cuáles sean dichos resultados, si lo que se busca es la igualdad en los mismos, es necesario invocar otro principio aristotélico: "desigual trato para los desiguales" (equidad vertical). Es decir, para alcanzar resultados homogéneos aparece como imprescindible actuar de manera heterogénea. En este sentido, Tedesco (2000) expresa que la equidad ya no puede referirse a tratar a todos por igual como se señalaba en el pasado, sino que se debe prestar atención y tratar a cada individuo de acuerdo a su situación.

Esto se relaciona con la noción de educabilidad presentada párrafos atrás. De acuerdo a la misma, habría individuos educables y no educables. Sin embargo, el criterio de la equidad vertical plantea la posibilidad de que todas las personas sean educables. López (2006) explica que detrás de la noción habitual de educabilidad existe un pacto tácito de distribución de responsabilidades entre la familia y la escuela; según este pacto a la familia le corresponde hacerse cargo de la primera educación sobre la que se basará la escolarización formal. Por ello, plantea la necesidad de un debate social que invite a estudiar cómo interferir en ese pacto para encontrar una manera de lograr que todos los individuos sean educables.

Cabe recordar que la noción de equidad conlleva implícito que para lograr la igualdad en un atributo puede ser necesario instaurar desigualdades en otros (Sen, 1999a). En el caso de la educación, la búsqueda de igualdad en los resultados implica la instauración de otras desigualdades a lo largo de la vida escolar de los individuos. Incluso, puede ocurrir que algunos individuos necesiten ingresar en el sistema educativo cronológicamente antes que otros (Llach, 2006).

Este criterio de equidad educativa basada en la igualdad de resultados es el que más se acerca a la lógica de las capacidades de Sen, ya que conlleva la idea de que todos los individuos puedan lograr cierto nivel de conocimientos y aptitudes que les 
otorguen herramientas para elegir el estilo de vida que desean vivir, dentro del cual se incluye hasta dónde desean llegar con su carrera formativa. Además, la igualdad en los resultados incorpora la idea de que no todas las personas pueden aprovechar de igual manera sus oportunidades, elemento primordial en el análisis de Sen.

En este sentido, si bien el enfoque de Sen no está basado en los resultados sino en la igualdad de oportunidades -producto de la igualdad en las capacidades de los individuos-, si se lograra verdaderamente igualar dichas capacidades, podría establecerse que los individuos en edad escolar llegarían todos a un mismo resultado educativo (aquel que es considerado esencial para que las personas dispongan de mayor libertad en el futuro).

$\mathrm{Si}$ todos los individuos en edad de egreso escolar alcanzaran un mismo resultado, esto querría decir que todos tuvieron acceso al sistema educativo, y que una vez allí sus diferencias en capacidades fueron compensadas. Es decir que, una vez dentro del sistema, cada uno pudo disponer de los recursos materiales (aulas, bancos, etc.) y no materiales (tiempo, apoyo psicológico, enseñanza, motivaciones, entre otros) necesarios para avanzar en su carrera escolar, ocurriendo que no todos recibieron lo mismo, ya que algunos necesitaron más que otros.

Sin embargo, en este punto es importante hacer una aclaración: puede ocurrir que el ámbito educativo no sea suficiente para lograr compensar las diferencias en las capacidades de origen de los individuos. Esto significa que quizás sea necesaria la aplicación de políticas vinculadas a otro ámbito para poder obtener el resultado deseado. Es de esperarse que, cuanto mayor sea el grado de desigualdad de origen, más limitada se encuentre la institución escolar para poder hacer frente a las compensaciones. Por ejemplo, es reconocida la importancia de las condiciones del hogar para el buen desempeño de los alumnos (Santos, 2007). Esto puede implicar la necesidad de llevar adelante políticas de vivienda y de alfabetización de adultos, entre otras cuestiones, para mejorar las capacidades de los individuos que habitan hogares con bajo clima educativo y características edilicias desfavorables para su desarrollo escolar.

De acuerdo a lo explicado en los últimos párrafos, el principio de equidad educativa basado en la igualdad en los resultados sería el más acorde a la propuesta de las capacidades de Sen. Por ello, en breve se analiza con mayor detalle el enfoque de la equidad educativa tomando como base de información los resultados.

\section{Equidad educativa: igualdad en los resultados}

Si bien el criterio de equidad educativa que rescata la igualdad en los resultados educativos presenta grandes problemas a la hora de definir políticas porque es difícil establecer cuáles son los resultados esperados y conocer la efectiva incidencia de los insumos ${ }^{11}$ y procesos en dichos resultados, aquí se elige dicha perspectiva para definir la equidad educativa a la luz del enfoque de Sen. Esto es así porque de los cuatro criterios presentados es el único consistente con el enfoque de las capabilities.

En este sentido, más allá de las limitaciones propias de la perspectiva en cuestión, cabe preguntarse qué significa igualdad en los resultados. ¿Quiere decir que todas las personas deben tener exactamente los mismos conocimientos? Ante la variedad existente de disciplinas ese objetivo sería imposible de cumplir. ¿Quiere decir que todos los individuos deben tener el mismo nivel educativo? Eso tampoco sería factible, ya que no todas las personas desean estudiar hasta el nivel máximo, que le otorgaría un título de doctor. ¿Qué significa entonces la igualdad en los resultados? López (2006) lo explica claramente:

El planteo de la igualdad en los resultados se traduce en un debate sobre cuál es el conjunto de conocimientos básicos que deben ser garantizados para todos, y a 
partir de los cuales cada uno desarrolla sus proyectos educativos individuales. En la perspectiva actual, un principio de equidad basado en la igualdad en los resultados apela a la igualdad en el acceso a un conjunto básico de conocimientos e igualdad de oportunidades para profundizar en la formación (p. 75).

En este sentido Bolivar (2005) señala que en educación básica, nivel que todas las personas necesitan adquirir para vivir plenamente sus vidas en sociedad, la equidad demanda igualdad en los resultados. Y también destaca la relevancia y la dificultad a la hora de definir el mínimo común que implica la educación básica.

Entonces, la cuestión pasa por establecer y consensuar cuál es el conjunto de conocimientos y competencias básicas. Este es un tema muy complejo y es necesaria la interacción de diferentes disciplinas para poder dar luz al debate social sobre este interrogante. No obstante, si bien alcanzar dicho consenso no es tarea sencilla, en América Latina existe cierto acuerdo en que la educación secundaria es primordial para el desarrollo social y para el destino personal de los individuos (Tedesco y López, 2002). Asimismo, se la plantea como horizonte de igualdad (López, 2006) y en varios países latinoamericanos es empíricamente identificado como el nivel educativo mínimo necesario para no caer en la pobreza (Calero, Escardíbul y Mediavilla, 2006).

En el nuevo contexto existente en América Latina, en el que los mercados laborales están cada vez más segmentados, donde existe un sector formal de alta productividad y un sector informal que produce para la subsistencia o los mercados locales, la probabilidad de ingresar al mercado formal está cada vez más asociada al conocimiento entendido como capacidad continua de aprendizaje. Si antes la educación primaria era suficiente para ingresar al mundo laboral industrial, ahora se requiere de un conjunto de competencias y saberes que se adquieren con más años de educación formal (López y Pereyra, 2004).

Por ello, se han llevado a cabo una serie de transformaciones en los países de la región, las cuales se han propuesto que la educación secundaria básica sea de buena calidad, lo cual incluye que brinde a los alumnos no solo la formación necesaria para su buen desempeño productivo, sino también para su desarrollo como ciudadano (Tedesco y López, 2002).

Además, la educación secundaria es el nivel educativo encargado de dar la posibilidad de alcanzar una cultura universal común, competencias comunicativas y de comprensión, facultades ligadas a la formalización y a la abstracción, capacidad de lectura y escritura, conocimientos históricos y geográficos universales, y un conjunto de actitudes y valores humanitarios (López y Pereyra, 2004).

En suma, se pretende que la educación media facilite que el alumno desarrolle habilidades, valores y actitudes que le permitan un buen desenvolvimiento en la sociedad. La enseñanza secundaria debe preparar para los estudios en el nivel superior y también para el mundo del trabajo para los que deseen insertarse en el mercado laboral. Asimismo, debe colaborar en la formación de la personalidad integral de los jóvenes, especialmente en los atributos que los ayudarán en su rol de ciudadanos. Por ello, la integración en la sociedad requiere que todos los individuos obtengan el nivel educativo medio. De lo contrario, aumenta su probabilidad de quedar excluidos o de solo acceder a una inclusión marcada por la pobreza y la vulnerabilidad. Por este motivo, en el debate de las políticas educativas aparece con mayor frecuencia el objetivo de lograr educación secundaria de calidad para todos (López y Pereyra, 2004). En Argentina, esto se vislumbra en el texto de la Ley de Educación Nacional (Ley No 26206):

La educación secundaria en todas sus modalidades y orientaciones tiene la finalidad de habilitar a los/as adolescentes y jóvenes para el ejercicio pleno de la ciudadanía, para el trabajo y para la continuación de estudios (Art. 30).

En este sentido, podría definirse la equidad educativa en torno al alcance del 
nivel medio. Sin embargo, eso solo haría hincapié en si las personas alcanzaron dicho nivel, pero no en cómo lo hicieron. Agregar a la definición de equidad educativa cuestiones en torno a la calidad de los resultados obtenidos por los individuos al pasar por el nivel medio se vuelve necesario. Es decir, no solo es relevante considerar cuántos individuos llegan a obtener el título secundario, sino también con qué conocimientos y competencias adquiridas.

En resumen, aquí se propone definir y cuantificar la equidad educativa tomando como base de información la igualdad en los resultados y lo explicado en los párrafos anteriores lleva a considerar específicamente la igualdad en los resultados en la educación media. Este esquema es congruente con la propuesta de Sen, ya que para que diferentes individuos alcancen los mismos resultados educativos se vuelve imprescindible tomar en cuenta las diferencias entre los mismos, es decir sus posibilidades heterogéneas de llevar adelante las oportunidades con las que se encuentran en su vida escolar. Esto implica que exista un trato diferencial, para lo cual es necesario instaurar desigualdades en diferentes momentos y aspectos de la vida educativa de las personas.

Sin embargo, podría decirse que definir equidad educativa con relación a los resultados de la educación media es definir equidad educativa básica, mientras que en realidad existe una forma aún más amplia de definir la equidad, incluyendo entre los objetivos de la educación media que esta provea a los alumnos la capacidad de elegir su futuro educativo con criterio y de manera fundamentada. Entonces, la definición de equidad se vuelve más compleja, ya que también incorpora la concepción de igualdad de oportunidades respecto a un nivel educativo superior (López, 2006).

Esta noción, más integral, se acerca aún más al concepto de equidad aportado por Sen, ya que se vincula en mayor medida a la posibilidad que tienen los individuos de tomar decisiones a lo largo de su vida y de elegir de esta manera su estilo de vida, al cual valorarán por diferentes motivos. López (2006) propone el ejemplo de dos personas que poseen un titulo secundario, una de ellas eligió no continuar sus estudios para dedicarse a los negocios, mientras que la segunda debió abandonar la idea debido a algún mecanismo de exclusión: arancelamiento, ausencia de oferta, costo de oportunidad, etc. En este ejemplo, si bien ambos individuos poseen el mismo título académico, no puede afirmarse que exista equidad educativa.

En síntesis, existirá equidad educativa global en una sociedad si todos sus miembros logran completar el nivel medio de enseñanza a la edad prevista, existiendo igualdad en los conocimientos y competencias que ellos han adquirido y, al mismo tiempo, todos pueden decidir libremente y sin ningún tipo de condicionamiento qué hacer con su carrera de formación en el futuro. Del mismo modo, Bolivar (2005) establece que en una primera fase de equidad educativa se requiere la igualdad en los resultados de la educación básica, mientras que una segunda fase el criterio podría definirse con relación a qué inequidades en otras esferas sociales (por ejemplo, la esfera de la riqueza) no interfieren en el desarrollo y las oportunidades de los alumnos.

Finalmente, cabe destacar nuevamente que la igualdad en los resultados no es incompatible con la igualdad en otros aspectos, sobre todo respecto a las oportunidades. Por el contrario, para que exista igualdad en los resultados es condición necesaria la igualdad en las oportunidades. Por ello, también es menester analizar diferentes cuestiones vinculadas al paso de los individuos por el sistema educativo.

Dado que la escolarización es un proceso continuo, la igualdad debe analizarse en cuanto al acceso, la supervivencia (probabilidad de permanecer en el sistema educativo), los resultados o output (definidos como igualitarios cuando cualquier alumno, sin importar su condición social de origen, tiene la probabilidad de aprender 
las mismas cosas que el resto) y los beneficios de esos resultados (outcome), es decir, las oportunidades sociales fruto de la etapa de escolarización (Calero, 1999; Feijoo, 2002; Bolivar, 2005). En este sentido, Sen (1999a) resalta que estas no se vinculan únicamente al mercado laboral, sino que también tienen que ver con la capacidad de los individuos para desarrollarse como ciudadanos, educar a sus hijos y vivir integralmente.

\section{Conclusiones}

A lo largo de este trabajo se ha realizado un exhaustivo análisis del concepto de equidad y de su aplicación en el ámbito educativo. Ha quedado manifiesto que es un concepto ambiguo y no es sencillo de definir. Por lo tanto, es necesario que quien lo utilice haga explícito cuál es el criterio que está tomando en cuenta al hacerlo, ya que la consideración de diferentes bases de información puede llevar a resultados y conclusiones muy disímiles.

La educación es un elemento esencial en la vida de las personas. Las personas más educadas pueden integrarse más fácilmente a la sociedad en la que viven, tanto a nivel político-ciudadano, como productivo. Este hecho se debe a que la educación permite al individuo invocar sus derechos, intercambiar ideas, obtener un empleo, cuidar mejor su salud y la de su familia, entre otras cuestiones. Por estas razones, puede asegurarse que la educación ayuda a aumentar las libertades individuales.

En dicho sentido, el enfoque de equidad propuesto por Sen, en el que el atributo a igualar son las capacidades, es sumamente apropiado para tratar temas vinculados a la educación. Ese es el primer motivo por el cual se ha elegido la perspectiva de Sen para definir equidad educativa en este trabajo. El segundo motivo tiene que ver con que es un enfoque que hace hincapié en que las personas son heterogéneas y a partir de allí se deriva que es posible prevenir aquellas inequidades provenientes de las diferencias en las capacidades de los individuos, cuestión no menor si se piensa que quienes se encuentran en edad de estudiar son niños y adolescentes. En tercer lugar, pero no por ello menos importante, cabe mencionar que se eligió este enfoque debido a que pone el acento en el humano, y no únicamente en su calidad de vida actual, sino también en sus potencialidades futuras, las cuales se vinculan estrechamente con la educación.

Luego, a la luz del punto de vista de Sen, se han analizado los criterios que históricamente han sido considerados para definir a la equidad educativa. A partir de allí se ha observado que el criterio más compatible con dicha perspectiva es el que propone la igualdad en los resultados educativos. De este modo, se ha derivado que el camino más apropiado para conceptualizar la equidad educativa en el contexto de las capabilities es definirla como la igualdad en las libertades fundamentales que adquieren los individuos a lo largo del sistema educativo.

La consideración de los resultados es más adecuada que las que proponen la igualdad en el acceso y/o en la metodología de enseñanza. En estos casos, se está afirmando que si todos acceden al sistema educativo y reciben el mismo tipo de enseñanza, deben lograr los mismos resultados y que, de no ser así, es responsabilidad de cada estudiante. Esta idea conlleva implícito el supuesto de que los alumnos son homogéneos y limita la posibilidad de aplicar políticas educativas tendientes a disminuir las brechas existentes entre las personas. Por el contrario, la idea de igualar los resultados entre los alumnos aparece como una mejor opción porque de ese modo se reconoce que existen discrepancias iniciales entre los mismos. Así se abre la posibilidad de debatir qué medidas sería necesario adoptar para equiparar dichas diferencias de origen y que todos logren alcanzar el mismo resultado final. 
En este sentido, es importante destacar la urgencia de que se plantee un diálogo social respecto al pacto implícito que existe en relación a las facultades de la familia y de la escuela: ¿pueden todas las familias brindar a sus hijos las competencias y condiciones necesarias para que articulen con el sistema educativo?; en caso de que dicha respuesta sea negativa, ¿qué puede hacer el resto de la sociedad para compensar dicha situación?, ¿qué políticas educativas pueden ser aplicadas por el Estado?, ¿qué otro tipo de políticas pueden volverse imprescindibles?

Otra cuestión que cabe señalar a la hora de hablar de igualdad en los resultados, es que debe tenerse en cuenta que los mismos incluyen cuestiones cuantitativas y cualitativas. De lo contrario, puede suceder que exista equidad en cantidad, pero no en calidad. En varios países de América Latina ha habido una mejora en cantidad, ya que ha aumentado el acceso al sistema educativo y la posibilidad de finalización del nivel medio, lo cual ha generado que aumente el porcentaje de personas con secundario completo. Sin embargo, para realmente hablar de equidad es necesario incorporar cuestiones vinculadas a la calidad, ya que puede suceder (y de hecho en Argentina sucede $^{12}$ ) que exista segmentación educativa y las competencias ligadas al título secundario varíen ampliamente entre colegios. Esto hace que algunas personas posean un título nominal, pero vacío de contenidos, mientras que otras tienen un título que realmente representa sus competencias, lo cual no es equitativo en término de resultados.

Por ello, en el trabajo se propone considerar que existe equidad en los resultados educativos si los individuos de una sociedad alcanzan a tener la cantidad de años de escolarización considerada necesaria para desarrollarse en su vida adulta (nivel medio), lo hacen a la edad correspondiente y con la misma calidad en las habilidades adquiridas. Entre ellas, la capacidad de elegir sobre su formación futura, es decir la posibilidad de tener igualdad en la oportunidad de optar por cursar el nivel educativo superior.
En síntesis, la manera en que se definen conceptos controvertidos, como se ha visto aquí que son la equidad en general y la equidad educativa en particular, no es trivial. Detrás de cada definición hay supuestos implícitos y las conclusiones a las que se llega son diferentes. Por ende, también son diferentes las recomendaciones de política que pueden surgir a partir de las mismas. En este trabajo, y por los motivos ya expuestos, se ha definido equidad educativa como igualdad en las capacidades adquiridas por las personas que han logrado cursar el nivel medio. Esto supone implícitamente que las personas son diferentes y que desde la política educativa (o de otro tipo) pueden (y deben) llevarse a cabo acciones que busquen compensar las diferencias de origen.

\section{Notas}

$1 \quad$ El autor utiliza la palabra prácticas para hacer referencia a cualquier forma de actividad presente en un sistema de reglas que define roles, premios, defensas, etc.

2 Así, el cálculo utilitarista se ve distorsionado cuando se aplica a personas que se encuentran en una condición de carencia crónica y han aprendido a obtener un placer máximo de gratificaciones mínimas o en el caso de individuos empobrecidos que, bajo una creencia religiosa de que son los elegidos de Dios, encuentran felicidad en una situación de miseria.

3 Roemer no hace hincapié en cuál es el resultado a medir aunque plantea que puede ser el grado de éxito que tiene una persona en su vida.

4 Esto se explicará con mayor detalle en la sección siguiente.

5 A Rawls y Sen no se los incluye en esta clasificación porque, como ya se ha mencionado, no se focalizan ni realizan un análisis claro de la responsabilidad individual. Sin embargo, como se analizará en breve, puede considerarse que la propuesta de Sen debe incluirse en este grupo.

6 La cual, a su vez se vincula con las ideas a Arneson y Cohen (como ya se ha explicado anteriormente).

$7 \quad$ El "justice cut" representa el elemento que permite discriminar las cuestiones de las que es responsable el individuo de las que no (Igersheim, 2006). 
Para un análisis detallado de cada una de las libertades instrumentales ver Sen (1999a) "Desarrollo y Libertad". pp. 57-59. Berlin (1974, citado en Sen 1999b) explica que la libertad puede entenderse como libertad positiva o negativa. La primera se refiere a la libertad que tiene una persona para hacer algo, teniendo en cuenta lo que dicha persona puede llegar a realizar, sin considerar restricciones externas. La libertad negativa tiene que ver con las limitaciones que le son impuestas a un individuo por parte de otros o del gobierno. Por ejemplo, si alguien no puede caminar por el parque porque es paralítico, va en detrimento de su libertad positiva. En cambio, si esa misma persona no puede pasear por el parque, pero no porque es minusválido, sino porque sería muy peligroso por la posibilidad de que lo ataquen criminales, se estaría violando su libertad negativa (además de la positiva).

Para mayor detalle sobre las características de los alumnos hoy esperadas por el sistema educativo ver "Equidad educativa y desigualdad social”, pp. 81-91 (López, 2006).

En el ámbito de la economía de la educación, se denomina insumos a todos los recursos (tangibles e intangibles) necesarios para que un individuo adquiera capacidades en el proceso de enseñanza.

Ver "Equidad educativa"

\section{Referencias bibliográficas}

Aghion P., y Howitt, P. (1998). Endogenous Growth Theory. Ed. MIT Press. Cambridge.

Akerlof, G. y Kranton, R. (2002). "Identities and schooling: Some lessons for the economics of education". Journal of Economic Literature, 40 (4). Pp 1167-1201.

Armesto, A. (2007). "Teorías de la justicia: ¿utilidad, igualdad o mérito?" Palinurus: Engaging Political Philosophy $N^{o}$ 12. Journal electrónico disponible en http://anselmocarranco.tripod.com/id60.html.

Arneson, R. (1989). "Equality and Equal Opportunity for Welfare". Philosophical Studies 56 (1). Pp. 77-93.

De Azcárate, P. (1874). Obras de Aristóteles, puestas en lengua castellana Moral a
Nicómano. Libro V. Capítulo primero. Disponible en http://www.filosofia.org/cla/ari/azc01119.htm.

Barham V. y Boadway R., Marchand M. y Pestieau P. (1995). "Education and the poverty trap". European Economic Review $N^{\circ}$ 39. pp: 1257-1275.

Birdsall, N. y Lodoño, J. (1997). “Asset Inequality Matters: An Assessment of the World Bank's Approach to Poverty Reduction". The American Economic Review, Vol. 87, No. 2. Pp.32-37.

Bolivar, A. (2005). "Equidad educativa y teorías de la justicia". Revista electrónica iberoamericana sobre calidad, eficacia y cambio en educación. Vol. 3. $N^{\circ}$ 2. pp. 42

Caballero, J. (2006). "La teoría de la justicia de John Rawls" Ibero Forum. Voces y contexto. Año 1. N.II. Pp. 1-22.

Calero, J. (1999). "Indicadores de equidad interna y externa en la educación superior. Metodologías y una aplicación al caso español". Ponencia presentada en el Seminario "Indicadores universitarios: tendencias y experiencias internacionales", Ministerio de Cultura y Educación, Secretaría de Políticas Universitarias, Buenos Aires.

Calero, J., Escardíbul, J. y Mediavilla, M. (2006). "Notas para la construcción de un sistema de indicadores sobre desigualdad y educación en América latina". Boletín de SITEAL, $N^{o} 5$. Buenos Aires: IIPE-UNESCO.

Castello, A. y Doménech, R. (2002). "Human capital inequality and economic growth: some new evidence". The Economic Journal, Vol. 112, No. 478, Conference Papers. pp. C187- C200.

Clune, W. (1994). "The Shift from Equity to Adequacy in School Finance". Educational Policy Vol. 8. $N^{o}$ 4. Pp. 376-394.

Cohen, G. (1989). "On the currency of egalitarian justice" Ethics. Vol. 99. $N^{\circ} 4$. Pp. 906-944. 
D’Lia, Y. y Maingon, T. (2004). "La equidad en el Desarrollo Humano: Estudio conceptual desde el enfoque de igualdad y diversidad". Documentos para la Discusión. Informes sobre Desarrollo Humano en Venezuela, del Programa de las Naciones Unidas para el Desarrollo (PNUD).

Dworkin, R. (1981). "What is equality?" Part 1: equality of welfare. Philosophy and public affairs. Vol. 10. $N^{\circ}$ 3. pp. 185-246.

Dworkin, R. (1981). "What is equality?" Part 2: equality of resources. Philosophy and public affairs. Vol. 10. $N^{o}$ 3. pp. 283-345.

Feijoo, M. (2002). Equidad social y educación en los años '90. Ed. IIPEUNESCO.

Fleurbaey, M.. (1995). "Equality and responsibility". European Economic Review $N^{\circ}$ 39. PP. 683-689.

Formichella, M. (2010). "Equidad educativa en la Argentina. Análisis desde la perspectiva de los resultados educativos". Libro electrónico de las XIX Jornadas de la Asociación de Economía de la Educación.

Gasparini L. (2001). Inequidad en el acceso a la educación secundaria y superior en la Argentina. Serie Fondo de Investigaciones. INDEC.

Guadagni, A. (2007). "Conferencia: los próximos 25 años". Reunión Anual de la Asociación Argentina de Economía Política (AAEP).

Hernández, A. (2006). La teoría ética de Amartya Sen. Ed. Siglo del hombre editores. Universidad de los Andes. Pp 136-148.

Honorable Cámara de Diputados y Senadores de la Nación. (2006). Ley $\mathrm{N}^{\circ} 26.206$.

Igersheim, H. (2006). A.K. Sen et J.E. Roemer: une même approche de la responsabilité? EconPapers: Working Papers of BETA 2006-08. Recuperado de http://econpapers.repec.org/paper/ ulpsbbeta/default2.htm
Llach, J. (2006). El desafio de la equidad educativa. Ed. Granica. Buenos Aires.

López, N. (2006). Equidad educativa y desigualdad social. Ed. IIPEUNESCO.

López, N. y Pereyra, A. (2004). "Educación media de calidad para todos, un difícil desafío para los países de América Latina". Boletín $N^{o} 6$ de SITEAL. Pp. 1-14.

López, R., Thomas, V. y Wang, Y. (1998). "Addressing the Education Puzzle. The Distribution of Education and Economic Reforms" Policy Research Working Papers. No. 2031. Washington, D.C.: World Bank.

Lucas, R. (1988). "On the mechanics of development planning" Journal of Monetary Economics. pp: 407-437.

Maldonado, S. y Ríos, V. (2006). "Más allá de la igualdad de oportunidades: Desigualdad de ingresos, responsabilidad individual y movilidad social en el Perú”. Recuperado de http:// www.cies.org.pe/investigaciones/ pobreza/igualdad-oportunidades.

Mendicoa, G. y Veneranda, L. (1999). Exclusión y marginación social. Nuevas perspectivas para su estudio. Ed. Secretaría de desarrollo social. Espacio Editorial. Buenos Aires.

Molina, M. (2000). "Estudio sobre criterios para la evaluación de la justicia social en las instituciones educativas" Biblioteca Digital de la OEI. Evaluación de la educación. Disponible en www.oei.es/oeivirt/ evaleduc.htm.

Montes, N. (2004). "Adolescentes y jóvenes en contexto. El marco cercano: la familia, y el marco amplio: los otros". En $L a$ trama de la desigualdad educativa. Compiladora: Guillermina Tiramonti. Ed. Manantial. Buenos Aires.

Morduchowicz, A. (2000a). "La equidad del gasto educativo: viejas desigualdades, diferentes perspectivas". Revista Iberoamericana de Educación $N^{o} 23$. pp: 165-186. 
Morduchowicz, A. (2003). Discusiones de economía de la educación. Ed. Losada.

Rawls, J. (1971). Teoría de la justicia. Ed. Fondo de Cultura Económica.

Rawls, J. (1999). "Justicia como equidad" Revista española de control externo Vol. 5, $N^{o} 13$, pp. 129-158.

Ribotta, S. (2006). Las desigualdades económicas. Un estudio desde el igualitarismo contemporáneo. Tesis correspondiente al Doctorado en Ciencia Jurídica: Teoría-HistoriaComparación. Universidad Carlos III de Madrid.

Roemer, J. (1998). "Igualdad de oportunidades" ISEGORIA Nro. 18. pp 71-87.

Roemer, J. (1995). "Equality and responsibility" Boston Review Forum, Social Equality and Personal Responsibility. Vol XX. Nro. 2. Recuperado de http:// bostonreview.net/dreader/series/ equality.html

Saito, M. (2003). “Amartya Sen's Capability Approach to Education: A Critical Exploration". Journal of Philosophy of Education, Vol. 37, No. 1. Pp. 17-33.

Santos, M. (2007). "Un modelo de trampa de pobreza con capital humano y calidad de la educación”. Anales de la Reunión Anual de la Asociación Argentina de Economía Política.

Sen, A. (1979). "Equality of what?" The tanner lecture of human values. Stanford University.

Sen, A. (1999a). Desarrollo y Libertad. Ed. Planeta.

Sen, A. (1999b). La libertad individual como compromiso social. Ed. Propuestas.

Sen, A. (2003). The importante of Basic education. Conferencia en Edinburgh University. Recuperado de http:// www.cis.ksu.edu/ ab/Miscellany/ basiced.html

Tedesco, J. (2000). Educar en la sociedad del conocimiento. Ed. Fondo de cultura económica.

Tedesco, J. y López, N. (2002). "Desafíos a la educación secundaria en América Latina". Revista de la CEPAL $N^{\circ} 76$. Pp. 55-69.

Thomas, V., Wang, Y. y Fan, X. (2002). “A new dataset on inequality in Education: Gini and Theil indices of schooling for 140 countries, 1960-2000”.

World Bank's paper. Recuperado de http:// www33.brinkster.com/yanwang2/ EducGini-revised10-25-02.pdf 